\title{
POLARIZATION OF LIGHT BY DUST IN GALAXIES
}

\author{
AINA ELVIUS \\ Stockholm Observatory, Saltsjöbaden, Sweden
}

\begin{abstract}
Several galaxies were observed for polarization during April and May 1972. Preliminary results are presented concerning those galaxies where polarization can be expected because of selective extinction or scattering of light by interstellar dust.
\end{abstract}

Dust clouds in other galaxies seem to be similar to the clouds in the Milky Way. Large-scale structures of the magnetic fields related to the interstellar matter in most types of galaxies may be revealed by observations of the polarization of light from such galaxies. Some observations of this kind have been made earlier.

Most of the observations were made with the 72-in. Perkins reflector of the Ohio Wesleyan and the Ohio State University at the Lowell Observatory. Additional data were obtained with the Lowell Observatory 42-in. telescope at the same site south of Flagstaff, Arizona. Dr J. S. Hall kindly let me use his area-scanning polarimeter (Hall, 1968) and the new computerized data-acquisition system built at the Lowell Observatory (Albrecht et al., 1971).

Table I summarizes some data concerning the observed galaxies. Results of polarization measures in yellow light of NGC 4565 are illustrated in Figure 1. The polarization due to extinction in the dust clouds of the spiral structure projected against the center of the galaxy reaches $2.8 \pm 0.8 \%$ in the darkest part of the lane and is about $1 \%$ along most of the scan at the same side of the centre. The strongest electric vector is roughly parallel to the symmetry plane of NGC 4565 .

In blue light only a few scans were made of NGC 4565 and the results are less precise. The contribution of scattered light also seems to complicate the interpretation.

NGC 4216 is also a galaxy seen almost edge-on. It seems to differ from the type of NGC 4565, however, and the polarization is also different. There is a weak polarization at the bright side of the centre which might be due to scattering of light by particles aligned in a magnetic field along the spiral arms. There is some doubt whether the effect is real, because the degree of polarization is only 2 or 3 times the error expected from pulse count statistics.

NGC 4438 was included in the observing list mainly because of the dust clouds ejected toward the north-east under the influence from a nearby $E$ galaxy. The light from these clouds is very faint and the data uncertain. No remarkable polarization was found in these faint areas. In the bright part of the galaxy a polarization of $1.4 \%$ was recorded which is probably to be interpreted as interstellar polarization in the spiral arms of NGC 4438.

The peculiar galaxy NGC 3718 has a rather well defined 'bar' of dark matter through the nucleus and the main body. This dark lane does not look like normal 


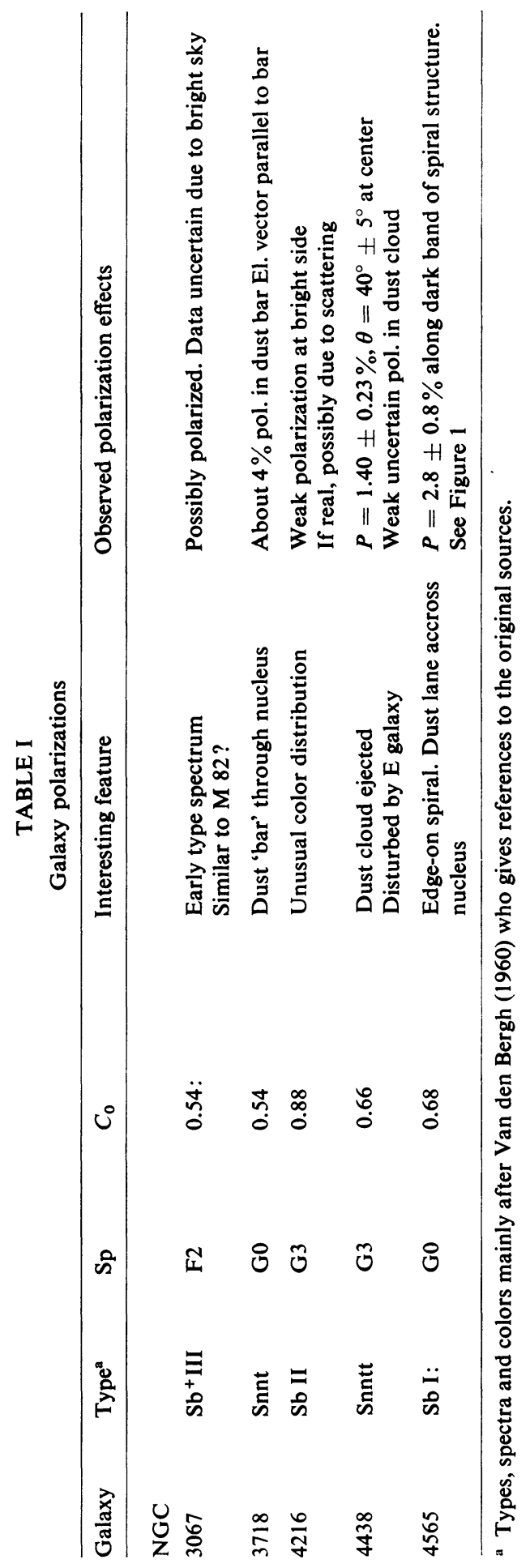




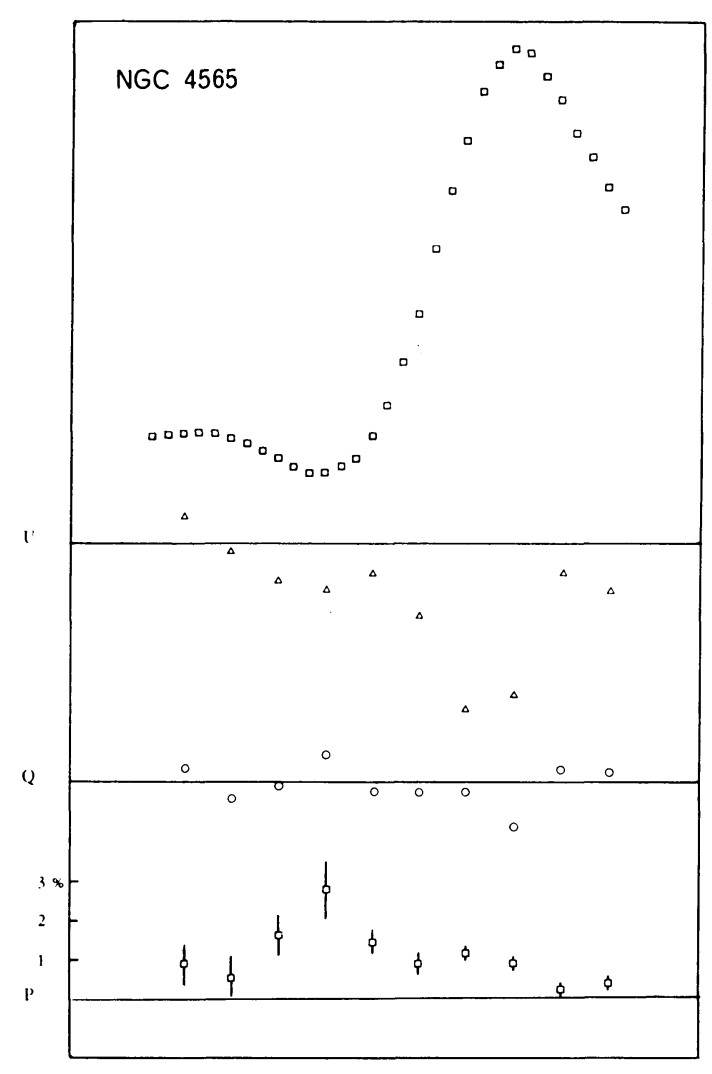

Fig. 1. Average data from several polarimeter scans with an OG 5 filter along the minor axis of NGC 4565. The top curve shows the distribution of yellow light along the scan. $U$ and $Q$ are Stokes' parameters. The bottom curve $P$ gives the polarization of light in per cent. Statistical errors from the pulse counts are indicated. $(Q$ and $U$ are given to indicate the direction of the polarization. Negative values of $U$ mean that the electric vector is approximately parallel to the major axis of NGC 4565 and thus also parallel to the dark lane.)

spiral structure. My observations show, however, that the light from the dark bar is polarized about $4 \%$ with the electric vector along the bar. This indicates that largescale magnetic fields are associated with the barlike structure. It may be speculated that this unusual dark bar may be the embryo of spiral arms formed by ejection of matter from the nucleus. It is not known to me whether NGC 3718 has been shown to emit radio radiation or to have an active nucleus.

The galaxy NGC 3067 may be a spiral but was classified as a ?I by Morgan (1958) who also published a photograph. This object was observed because of its possible similarity to M 82 which is known to show very high degrees of polarization in its outer faint regions due to scattering of light in dust clouds (Elvius, 1963, 1972; Sanders and Balamore, 1971; Visvanathan and Sandage, 1972). Preliminary reductions indicate the presence of polarization in NGC 3067 but the final conclusion must await a more careful study of the influence of a polarized sky background. 


\section{Acknowledgement}

This work was supported by a grant from the Swedish Natural Science Research Council.

Note added in proof. U. J. Schwarz has kindly informed me that high resolution observations in the $21 \mathrm{~cm}$ line have shown a strong concentration of neutral hydrogen along the bar (dust-lane) of NGC 3718. It seems to rotate almost as a solid body. - The galaxy was detected by R. D. Ekers in continuum observations near $21 \mathrm{~cm}$ (including the hydrogen line emission).

\section{References}

Albrecht, R., Boyce, P., and Chastain, J.: 1971, Publ. Astron. Soc. Pacific 83, 683.

Elvius, A.: 1963, Lowell Obs. Bull. 5, 281.

Elvius, A.: 1972, Astron. Astrophys. 19, 193.

Hall, J. S.: 1968, Lowell Obs. Bull. 7, 61.

Morgan, W. W.: 1958, Publ. Astron. Soc. Pacific 70, 364.

Sanders, R. H. and Balamore, D. S.: 1971, Astrophys. J. 166, 7.

Van den Bergh, S.: 1960, Publ. David Dunlop Obs. 2, 159.

Visvanathan, N. and Sandage, A.: 1972, Astrophys. J. 176, 57. 